\title{
APNOEIC OXYGENATION IN THE MANAGEMENT OF A GLOTTIC FOREIGN BODY: A CASE IN POINT
}

\author{
E. K. Bhargava, E. W. Chan, P. Coyle, A. Qayyum \\ Peterborough City Hospital, United Kingdom
}

\section{INTRODUCTION}

- Aspirated glottic foreign bodies $\rightarrow$ a real challenge as far as the airway management is concerned

- Hazardous undertakings where successive laryngoscopy-hypoxaemia-re-oxygenation cycles can escalate to airway loss

- POINT: Peri-Operative Insufflatory Nasal Therapy $\rightarrow$ novel technique of apnoeic oxygenation $\rightarrow$ longer duration of apnoea $\rightarrow$ unhurried removal of the foreign body

\section{CASE}

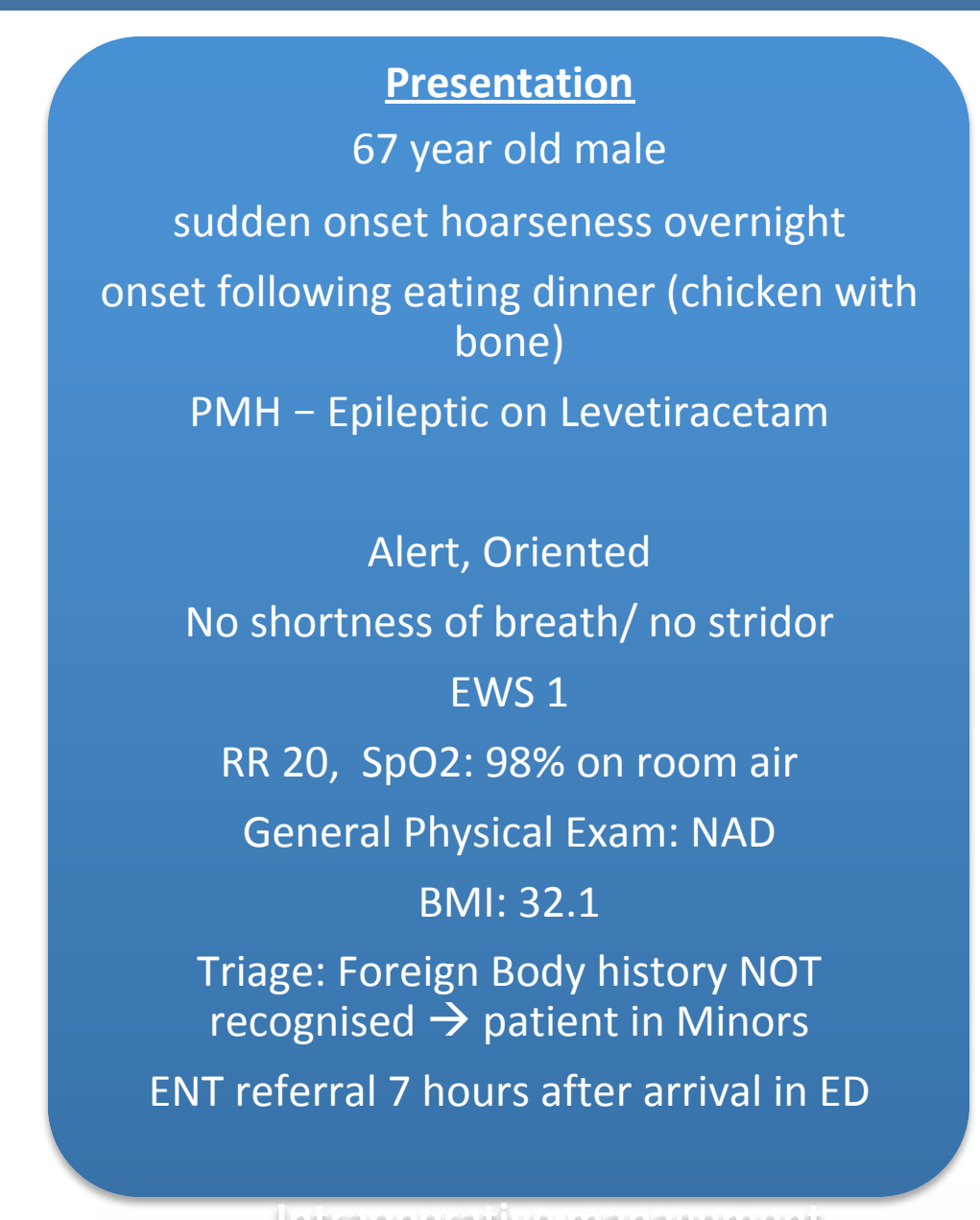

\section{No Theatre Free!}

ENT theatre anaesthetic room site of final treatment

Personnel: 2 ENT consultants, 2 Consultant Anaesthetists, ENT registrar, Theatre staff

Preoxygenation + apnoeic oxygenation using POINT

TIVA

Rigid Laryngoscopy: removal of glottic foreign body; arytenoids oedematous, TVC congested Maintained SpO2 99-100\% at 70-80 L/minute Total apnoeic time: 12 minutes
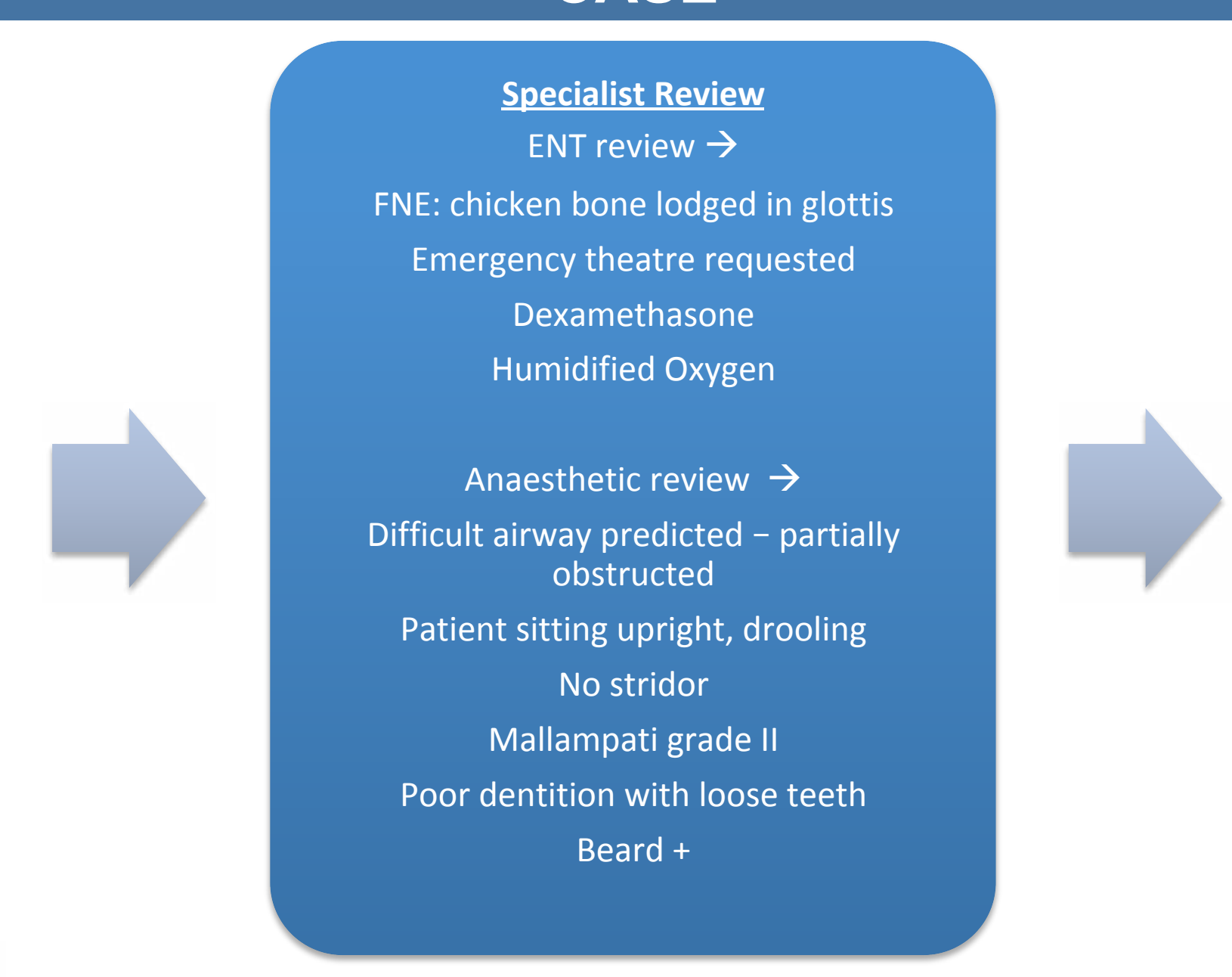

Airway Management Plan Issues:

Any form of intubation $\rightarrow$ high risk of displacing foreign body into trachea Jet ventilation $\rightarrow$ high risk of displacing foreign body into trachea Traditional Apnoeic oxygenation $\rightarrow$ short window for management before hypoxia and $\mathrm{CO} 2$ build-up
Proposed plan:
Plan A $\rightarrow$ Apnoeic oxygenation using POINT + Direct laryngoscopic removal Plan B $\rightarrow$ Ventilation Bronchoscopic

removal Plan C $\rightarrow$ Emergency Tracheostomy on standby

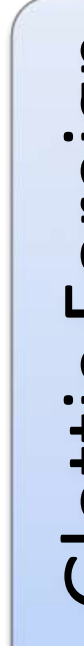

aphoeic time: 12 minutes

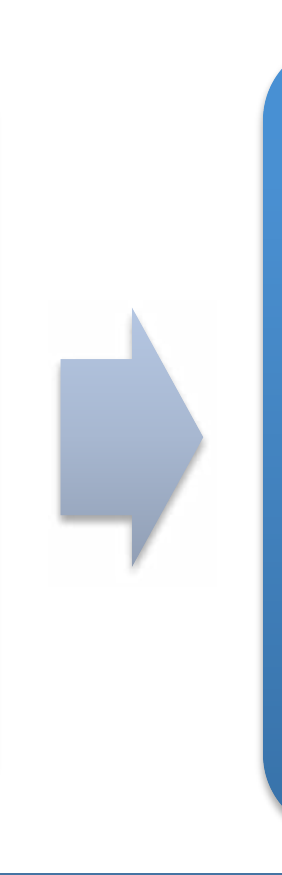

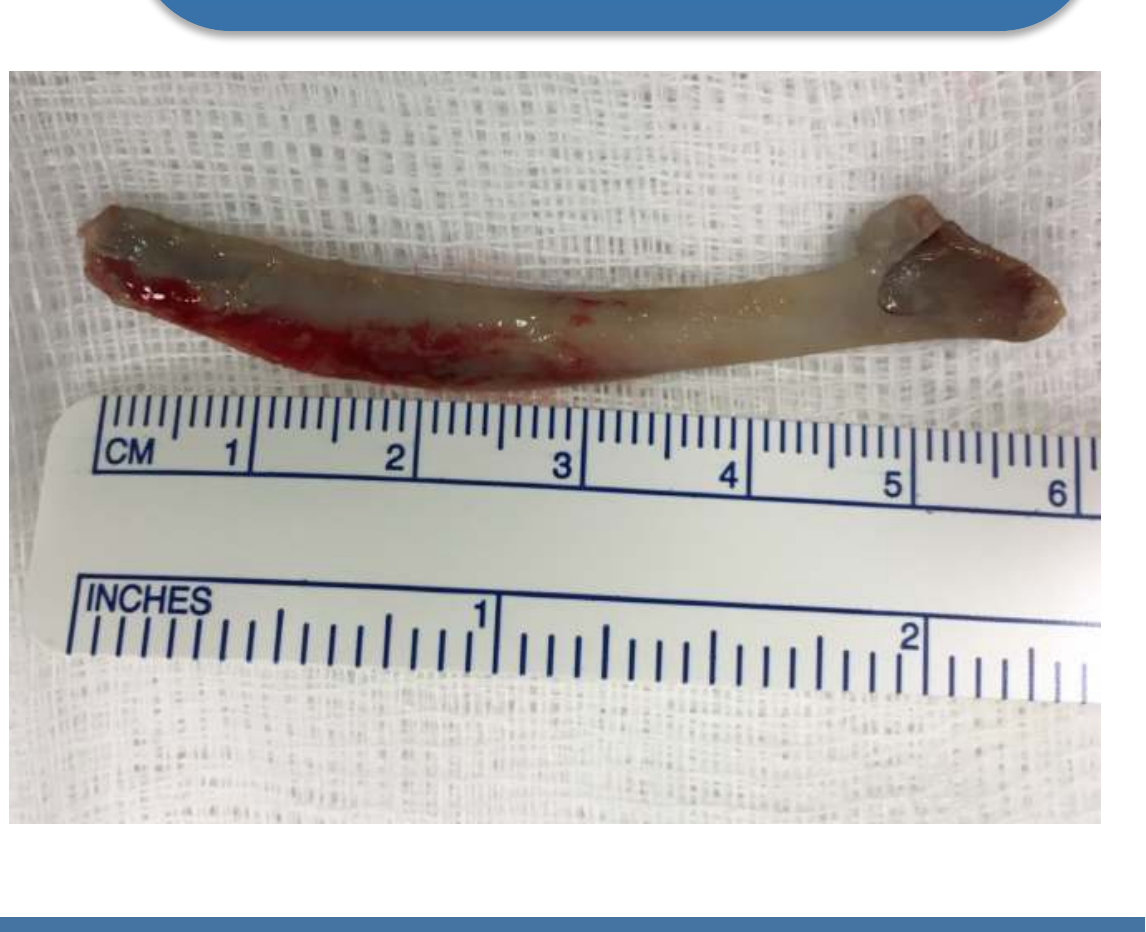

\section{DISCUSSION}

$\chi_{\text {- Large } \mathrm{FB} / \text { those with sharp edges }}$

- Usual presentation: wheezing, dyspnoea

- Presentation without airway symptoms:

rare

- If inhaled: coughing, stridor

- Rarely: sudden death due to glottic obstruction

- Timely intervention: KEY!

- Delays in diagnosis $\rightarrow$ complications (chronic wheeze, pneumonia, lung abscess, life-threatening respiratory obstruction)

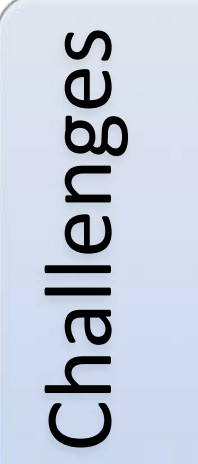

Human factors:

- Recognising glottic foreign body

- Involving specialists early

- Timely intervention

- Anticipating difficulties and planning ahead

Airway factors:

- intubation/jet ventilation not viable options due to risks of dislodgement

- Traditional apnoeic oxygenation: extremely limited window of action before toxic $\mathrm{PaCO} 2$ build up

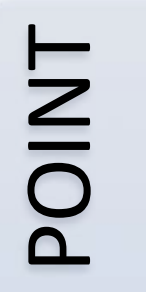

- PeriOperative Insufflatory Nasal Therapy

- Traditional technique of 'apnoeic

oxygenation' $\rightarrow$ respiratory acidosis due to $\mathrm{CO} 2$ build up

- Trans-nasal high flow humidified oxygen $\rightarrow$ aventilatory mass flow (traditional apnoeic technique to increase oxygen reserve) + flow-dependent ventilator exchange $\rightarrow$ prevents rise in $\mathrm{PaCO} 2$ while maintaining SPO2

- Longer safe duration of apnoea: up to 65 minutes

\section{LEARNING POINTS}

- Timely intervention is the key to prevention of life-threatening complications in managing glottic foreign bodies

- POINT allows for a long apnoeic window in cases where the airway cannot be secured $\rightarrow$ converting a pressured stop-start process to a smooth and unhurried undertaking $\rightarrow$ potentially reducing peri- and post-operative complications $\rightarrow$ preventing patient morbidity and mortality

\section{REFERENCES}

1. Sengupta S, Swaika S, Banerjee SS, Sheet J, Mandal A, Bisui B. Successful emergency airway management in a case of removal of foreign body bronchus in a pediatric patient. Anesthesia, Essays and Researches. 2014;8(2):250-252. doi:10.4103/0259-1162.134525.

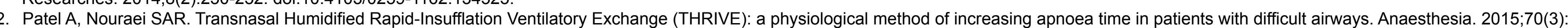
323-329. doi:10.1111/anae.12923.

3. Gupta K, Gupta PK. Laryngoscopic removal of unusual metallic foreign body of the subglottic region of the larynx. Anesthesia, Essays and Researches. 2010;4(2):106-108. doi:10.4103/0259-1162.73517. 